\title{
EVIDENCE OF A CORONA DISCHARGE INDUCED BY NATURAL HIGH VOLTAGE DUE TO VERTICAL POTENTIAL GRADIENT
}

\author{
D. HONG ${ }^{1 *}$, H. RABAT ${ }^{1}$, M.J. KIRKPATRICK ${ }^{2}$, E. ODIC ${ }^{2}$, N. MERBAHI ${ }^{3}$, \\ J. GIACOMONI ${ }^{4}$ AND O. EICHWALD ${ }^{3}$
}

\author{
${ }^{1}$ GREMI, UMR7344, Univ. of Orleans, CNRS, 45067 Orleans, France \\ ${ }^{2}$ GeePs | Group of electrical engineering - Paris, CNRS, CentraleSupélec, Univ. Paris-Sud, \\ Université Paris-Saclay, Sorbonne Université, 3 \& 11 rue Joliot-Curie, Plateau de Moulon 91192 Gif- \\ sur-Yvette CEDEX, France \\ ${ }^{3}$ LAPLACE, Univ. of Toulouse, UMR5213, 31062, Toulouse, France \\ ${ }^{4}$ AEROPHILE, 106 avenue Félix Faure, 75015 PARIS, France \\ *corresponding author: dunpin.hong@univ-orleans.fr
}

\begin{abstract}
This paper describes a study evidencing the creation of a corona discharge induced by natural high voltage found above the earth's surface due to the vertical potential gradient at an altitude of 125 meters. Experiments were made in fine weather in autumn and with little wind, on the Balloon Generali, a tethered helium balloon that takes tourists up for views above Paris. The measuring equipment was installed in the gondola suspended from the tethered balloon. A metal point electrode was also suspended from the balloon at the same level as the gondola at a lateral distance of $8 \mathrm{~m}$. During the ascent, as soon as the altitude of the gondola exceeded about $65 \mathrm{~m}$, a current coming from the metal point electrode was detected. The value of this current fluctuated but generally increased with height and reached an intensity of up to $320 \mathrm{nA}$ at $125 \mathrm{~m}$. When the electrode tip approached the gondola, which was electrically grounded via the pull cable, the current was lower; when the tip was replaced by a larger rounded electrode, the current was observed to disappear. The combination of these observations clearly demonstrated the presence of a corona discharge caused by the vertical potential gradient, and in turn, the existence of a corona discharge without high voltage power supply evidenced the obtaining of a natural high voltage.
\end{abstract}

\section{INTRODUCTION}

In fair weather, due to the so-called global atmospheric circuit, the surface of the Earth is negatively charged with respect to the positively charged atmosphere. An electric field, inherent to the so-called vertical potential gradients (VPG), directed generally towards the ground, is then present in the air of the troposphere, and its value varies according to the season, the weather and other parameters; inversion of the field occurs during thunderstorms, completing the circuit. With a typical value of $100 \mathrm{~V} / \mathrm{m}$ according to a well-known textbook [1], the potential difference between two points having an altitude difference of $100 \mathrm{~m}$ is then $10 \mathrm{kV}$. This is a voltage high enough to lead to the initiation of a corona discharge with a point electrode in order to collect the electrical charge in the atmosphere as attempted by Benjamin Franklin and Thomas-François Dalibard. Indeed, studies of electrical phenomena in the atmosphere date back to Benjamin Franklin who suggested attempting to collect electricity from thunderclouds by means of a point on a high tower or steeple and insulated from the ground as described by J.A. Chalmers in his monograph entitled "Atmospheric Electricity" [2]. In this book, Chalmers also reported many studies on "discharges from Points Attached to kites and balloons" [3-8]. For instance, Kirkman and Chalmers [3] used a point at 
about $30 \mathrm{~m}$, which is considerably higher than the neighboring trees; they found that point discharge occurred for potential gradients down to $250 \mathrm{~V} / \mathrm{m}$ and that the current depended on the wind speed.

Motivated by the collection of electric charge and the exploitation of the natural vertical potential gradient, several studies have been conducted, including that of Plauson who carried out experiments with a balloon made of aluminum leaf with collecting needles of amalgamated zinc and a radium preparation as an ionizer [9]. This motivation continues as testified by recent patents [10-12].

The present study was performed to examine the possibility of obtaining an induced corona discharge due to natural high voltage induced by the VPG by measuring its mean current. The following section describes the experimental setup of the experiment, as well as the apparatus for measuring the mean current of the corona discharge. Section 3 presents both numerical simulations and measurement results and discussions. A conclusion completes this paper.

\section{EXPERIMENTAL SETUP}

This study was conducted thanks to the Balloon of Paris, a tethered balloon intended for tourism (figure 1-a). This balloon rises up to $150 \mathrm{~m}$ in less than three minutes and recently, in fine weather, it rose up to $300 \mathrm{~m}$. The balloon has been used since 2012 to measure fine particulate matter in the atmosphere southwest of Paris to a height of $125 \mathrm{~m} \mathrm{[13]} \mathrm{and} \mathrm{the}$ company offered opportunities for scientific experiments.

Experiments were made in autumn in fine weather with little wind. The measuring instruments, including an oscilloscope (Lecroy Waverunner 62Xi, $600 \mathrm{MHz}$ ), a high voltage probe (Lecroy, PMK-14KVAC), were installed in the gondola suspended from the balloon $25 \mathrm{~m}$ below the top of the balloon. The oscilloscope was powered by a small generator on board the balloon.
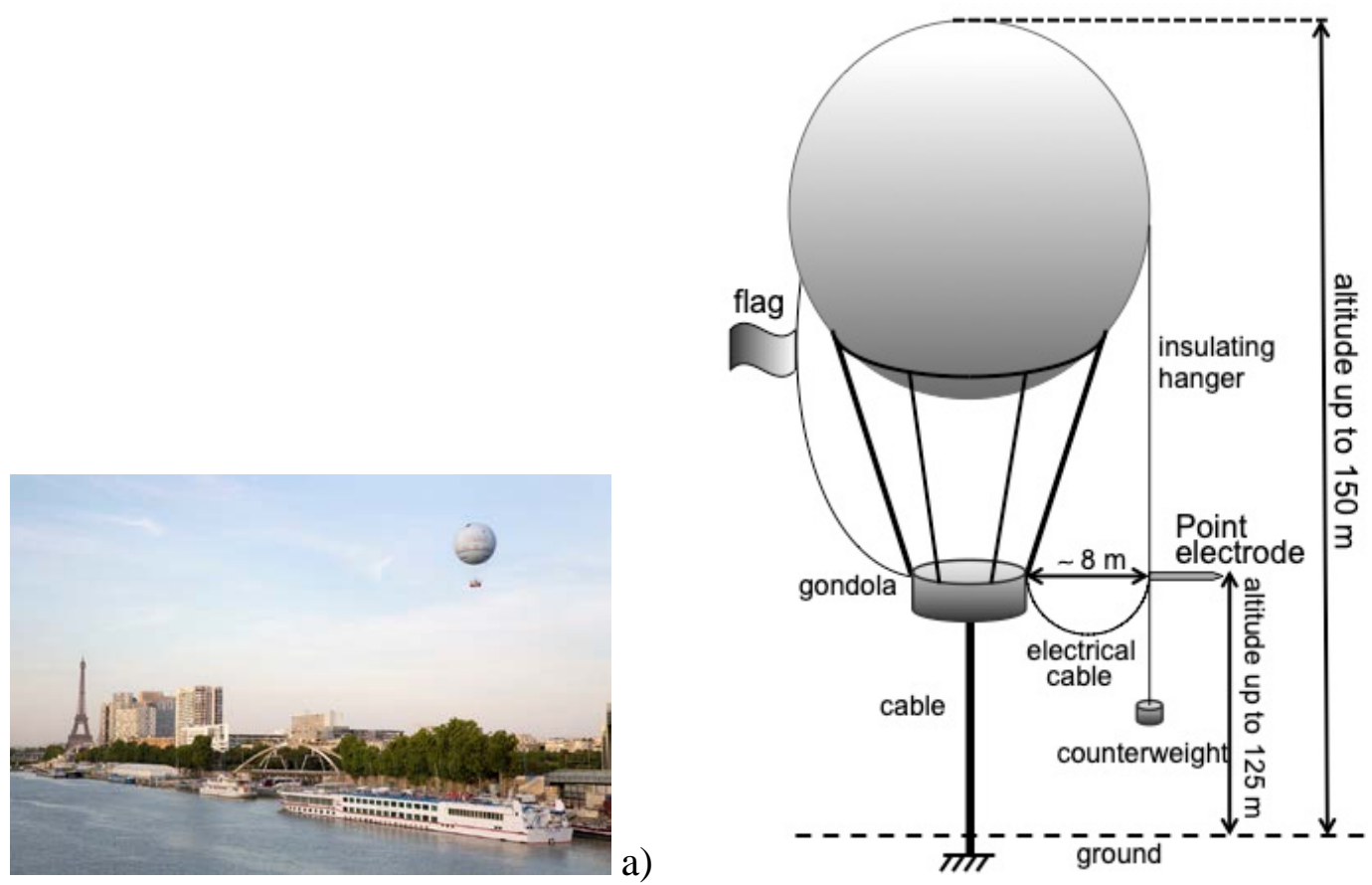

b)

Fig. 1: a) Picture of the balloon in the sky of Paris. b) Experimental set-up. In calm weather, the balloon can rise up to $300 \mathrm{~m}$. The electrical cable connecting the point electrode is sheathed to avoid unwanted electrical contact. 
As shown in figure 1-b, the gondola rises up to an altitude of $125 \mathrm{~m}$ when the top of the balloon at $150 \mathrm{~m}$ in altitude. If the electrical field is $100 \mathrm{~V} / \mathrm{m}$, the potential at $125 \mathrm{~m}$ should be $12.5 \mathrm{kV}$ with the land as potential reference. As the gondola is electrically connected to the ground via the pull cable, its presence at $125 \mathrm{~m}$ greatly disrupts the surrounding equipotential lines, as shown in figure 2. This numerical calculation was performed with the commercial $\mathrm{COMSOL}^{\circledR}$ software using the $2 \mathrm{D}$ axis-symmetry approximation assuming a homogeneous electric field of - $100 \mathrm{~V} / \mathrm{m}$ (see section 3.1 for more calculating detail).

According to figure 2, a higher electric field will be present in the lateral direction away from the gondola. Therefore, a point electrode was placed at a distance of about $8 \mathrm{~m}$ from the gondola. This metal tip with a $25 \mu \mathrm{m}$ radius of curvature was suspended using a weighted nylon rope from the balloon at the same altitude as the gondola. The point electrode was electrically connected via a current collecting capacitor (see Fig. 3), to the grounded body of the gondola. This leads to a strong difference in potential between the tip and its surroundings, creating the conditions for the occurrence of a corona discharge as shown by the numerical calculations in section 3.1.

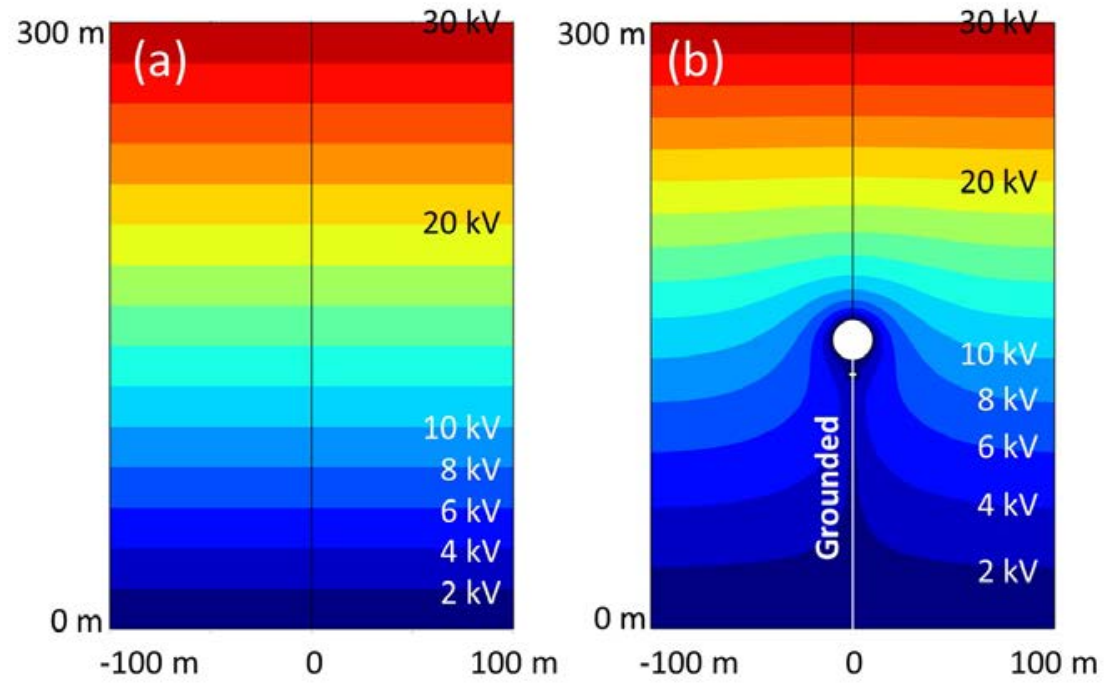

Fig. 2: Illustration of the equipotential lines without (a) and with (b) the presence of a grounded gondola and balloon.

Because the tip has a lower potential, the corona discharge must be negative, which produces Trichel pulses [14]. A 2 GHz bandwidth current probe (Tektronix CT-1, 5mV/mA) was used to detect such Trichel pulses. However, it was not possible to detect these pulses due to electromagnetic noise on the channels of the oscilloscope caused by the gasoline generator used to supply the oscilloscope which exceeded the expected amplitude from a Trichel pulse. To measure the average current of the corona discharge, a simple device was used consisting of the insertion of a $2 \mathrm{nF}$ capacitor between the point electrode and the grounded body of the gondola, together with the continuous measurement of the capacitor voltage using a high voltage probe (Lecroy, PMK-14KVAC) as shown in figure 3. The resistance $R$ in this circuit represents the input impedance of the voltage probe, which was $100 \mathrm{M} \Omega$. This device was previously tested and validated in the laboratory in the following manner: a stable corona discharge created by using a high voltage power supply, was used, and drift charges were collected by the counter electrode, which was a metal plate placed in front of the point electrode. The collected charges charged the capacitor to a stable voltage named $V_{C}$. When the voltage is stable, i.e., the capacitor is no longer being increasingly charged by the collected 
current, this means that the full collected current passes through the resistance $R$, whose voltage is also $V_{C}$. The average corona discharge current is thus $V_{C} / R$.

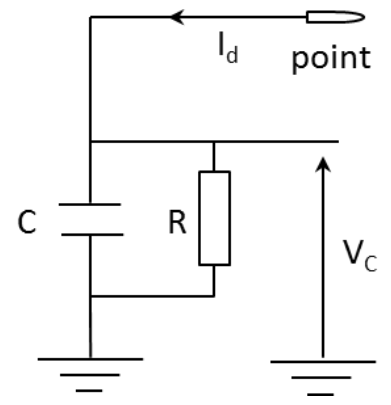

Fig. 3: Mean current measurement device. $R$ represents the input impedance of the voltage probe.

The balloon usually rises at a speed of $48 \mathrm{~m}$ per minute and descends at a speed of $36 \mathrm{~m}$ per minute. The time constant of the balloon movement is much larger than the time constant of this RC circuit, which is $0.2 \mathrm{~s}$, so the electrical measurements can be considered as in steady state. Therefore, the measurement of the voltage gave the mean collected current and the nonzero current is interpreted as evidence for the existence of a corona discharge.

\section{RESULTS AND DISCUSSION}

\subsection{Numerical calculation of the electrical field near a point electrode}

It is well known that the critical electric field in air (above which ionization and detachment becomes higher than attachment processes) at atmospheric pressure is about $30 \mathrm{kV} / \mathrm{cm}$ (i.e. $3 \mathrm{MV} / \mathrm{m}$ ) [15]. In order to check if there was chance to obtain electrical breakdown at the tip to ignite a corona discharge, calculation of electrical field near the point was done using the commercial COMSOL ${ }^{\circledR}$ software. The main strategy used to estimate this electric field is described hereafter. The challenge is to estimate the electric field around a tip of $25 \mu \mathrm{m}$ in radius of curvature suspended at $125 \mathrm{~m}$ above earth as shown in figure 1-b. Strictly speaking, the problem should be solved in a 3D complex geometry with a spatial scale ranging from micrometers (in order to properly define the shape of the tip) to a few hundred of meters. Obviously, such a simulation would consume huge memory and computing times. To overcome these difficulties, the calculation was done in two steps. In the first step, the atmospheric electric field was simulated using a 2D axis-symmetric domain without and with the gondola and balloon. The chosen geometry, dimension and boundary conditions are shown in figure 4 . When the gondola, the balloon are added to the simulation, it is assumed that they are grounded via the pull cable. The obtained results were already shown in figure 2 and discussed in the previous section. Figure 5 shows a zoom of figure 2-b in the region where the point electrode was positioned in order to collect the current.
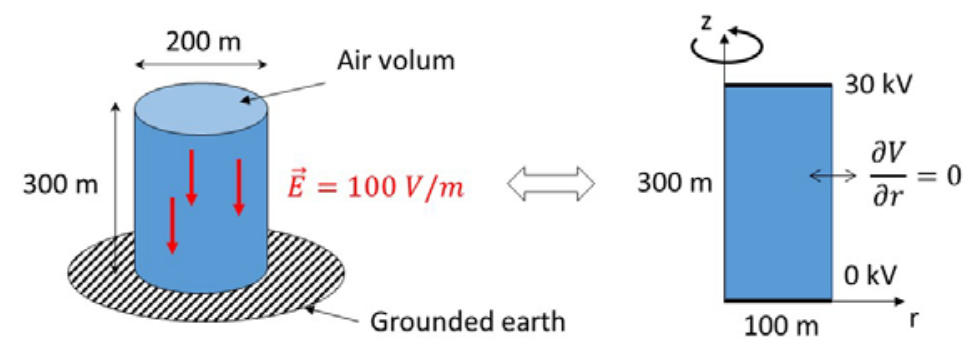

Fig. 4: The chosen geometry and the equivalent 2D axis-symmetry used in our first step simulation conditions. 
The second step in the simulation consisted of calculating the electric field in a much smaller region in the vicinity of the point electrode. This was done by placing the point electrode and the wire connecting it to the grounded gondola inside a horizontal cylinder domain (whose borders are drawn with dashed white lines in figure 5: wire and electrode are schematically represented by the black arrow). In this context, the first simulation results allow for the estimation of the value of electric potential used for the boundary condition to be used at the right edge of the second simulation domain. In addition, the left hand side of this new domain was grounded and zero Neumann boundary conditions were fixed along the lateral borders of the horizontal cylindrical domain. The boundary condition for the potential on the right hand side was chosen to be equal to $5 \mathrm{kV}$ with reference to the potential profile obtained in figure 5 at four meters from the tip location. Here, it is worth noting that it was assumed that this distance $(4 \mathrm{~m})$ was far enough so that the addition of the small grounded electrode had a negligible effect on the boundary potential value calculated without the electrode and wire. In reality, the presence of the grounded electrode and the wire connecting it to the gondola will perturb the electric field just as the balloon itself has been shown to do. Even if our chosen conditions do not exactly reproduce the potential profile inside the dashed white domain obtained by the first step calculation, it is here assumed that the simulation was representative of the initial electrical environment in which the electrode was placed.

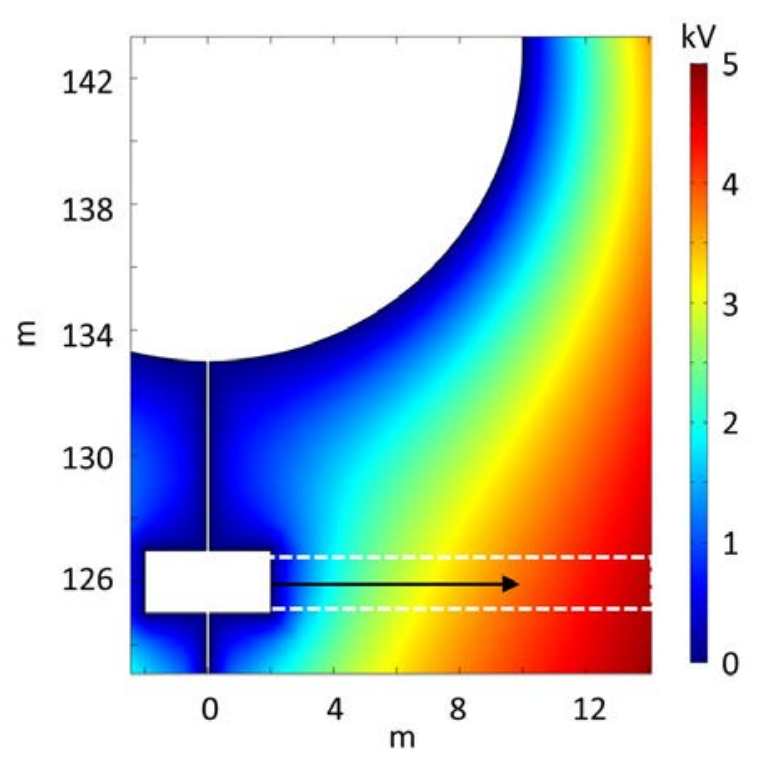

Figure 5: Zoom of figure 2b near the gondola. The white dashed area defines the new simulation domain in which the pin connected to the gondola (black arrow) was taken into account.

It was therefore possible to take into account the electrode and to estimate its effect on the repartition of the electric potential. In the simulation conditions, the conductive wire that connected the electrode to the gondola had a diameter of $1 \mathrm{~cm}$ and is $8 \mathrm{~m}$ in length. The electrode had a diameter of $1 \mathrm{~mm}$ and was $5 \mathrm{~cm}$ in length. The tip of the electrode presented a conic shape and ended in a sphere of $25 \mu \mathrm{m}$ radius. As can be seen on figure 6-a, the surrounding equipotential lines were greatly disrupted when the tip was added and the highest potential gradient was located at the right-hand side of the tip. Consequently, the electric field around the pin becomes very high and reaches a maximum of $200 \mathrm{kV} / \mathrm{cm}$ on its spherical edge. Figure 6-b shows the electric field profile around the tip with a legend scale cut off at $30 \mathrm{kV} / \mathrm{cm}$. Therefore, the red zone around the tip shows the volume where ionizations and detachments are the dominant processes, meaning that corona discharge may be initiated at least inside this volume. In fact, the volume can be extended because of the diffusion of for 
instance (i) negative ions whose detachment threshold voltage is lower than $30 \mathrm{kV} / \mathrm{cm}$ and (ii) metastable species that present a much lower ionisation threshold energy than species in their ground state.
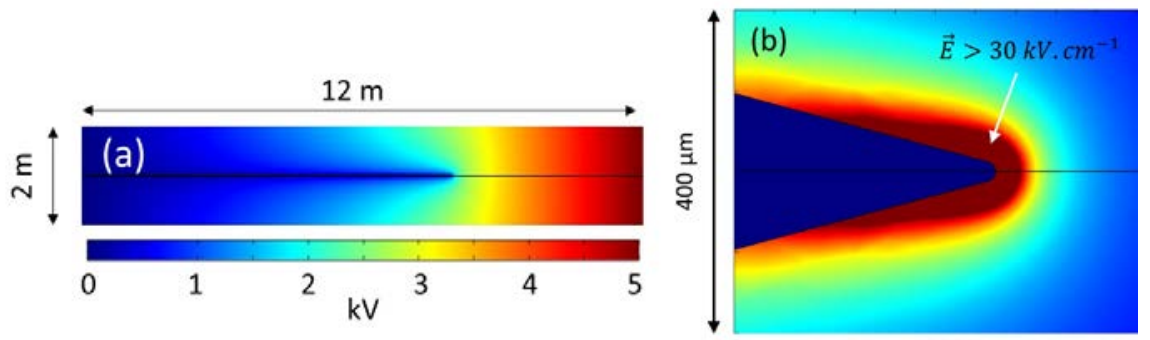

Figure 6: (a) Potential profile in the dashed white domain of figure 5 when the pin is connected to the gondola (see the text for more details) (b) Zoom of the electric field magnitude around the pin.

As the tip was grounded, the corona discharge was a negative one; electrons and negative ions left the point, then the zone, while positive ions accelerated by the field bombarded the tip creating other electrons by secondary emission processes leading to a sustained discharge which was probably pulsatile due to Trichel pulses.

\subsection{Current measurement during balloon ascent}

During an ascent, the voltage of the capacitor as a function of time was measured by the oscilloscope. The voltage was positive, which means that the direction of the current indicated in Fig. 3 is correct and that electrons leave the point electrode as discussed in section 3.1. It is therefore a negative corona discharge as expected (see discussion in section 2). Using the simple calculation explained in section 2, it is possible to determine the current as a function of time. Knowing the speed of ascent, it is then possible to express current as a function of the altitude as shown in figure 7-a. However, the altitude values given here are approximate because the speed of ascent controlled by an operator can vary by up to $15 \%$, and the take-off time has an uncertainty of several seconds.
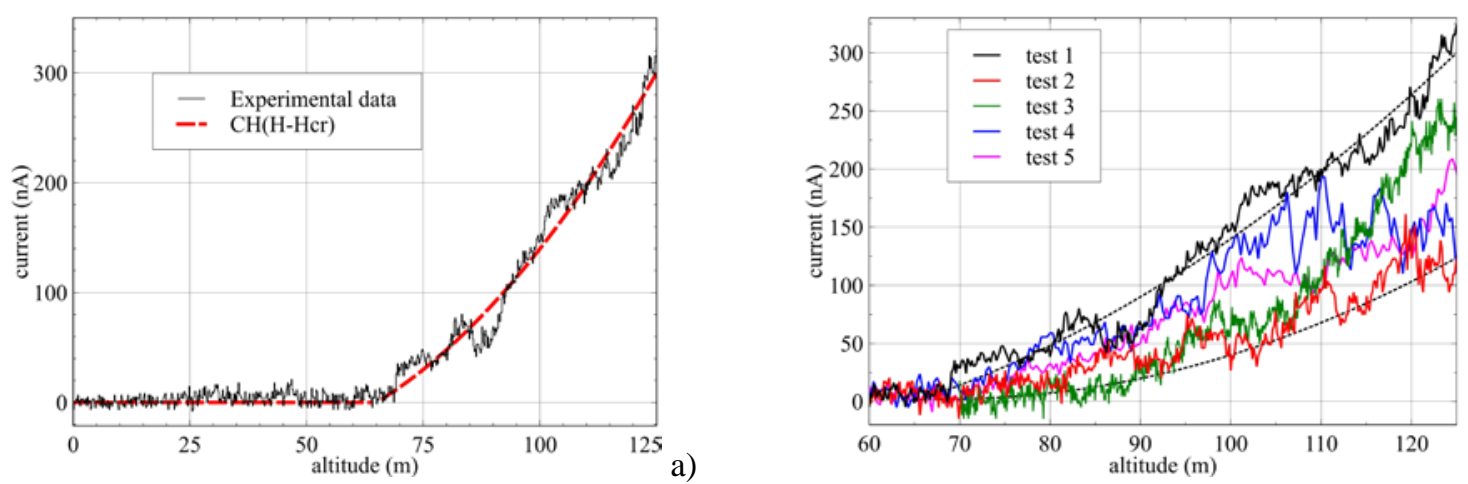

Fig. 7: (a) Collected current as a function of altitude. The balloon rises with an approximate speed of $48 \mathrm{~m}$ per minute. The experimental current may be fit to with the one calculated by using $\mathrm{i}=\mathrm{C} \mathrm{H}(\mathrm{H}-65)$ by adjusting the value of the constant C. (b) Collected current as a function of altitude for 5 tests.

It can be seen that current appeared when the gondola had exceeded a threshold in altitude of approximately $65 \mathrm{~m}$. It can be deduced that, from this threshold, the difference in potential between the tip and its surroundings is large enough to initiate a corona discharge. Considering the experiment reported by the reference [3], in which a discharge was obtained at an altitude of $30 \mathrm{~m}$ and the electrical field was stated to be $250 \mathrm{~V} / \mathrm{m}$, these two values imply a potential difference of $7.5 \mathrm{kV}$. Coupling this potential difference to the altitude threshold value of $65 \mathrm{~m}$ in this work yielded an electrical field of $115 \mathrm{~V} / \mathrm{m}$. 
The curve indicated that the discharge was more intense with increasing altitude, therefore with a greater difference of potential. Generally speaking, the current of a corona discharge is given by $\mathrm{i}=\mathrm{C} \mathrm{V}\left(\mathrm{V}-\mathrm{V}_{\mathrm{CR}}\right)$ where $\mathrm{C}$ is a constant, $\mathrm{V}$ is applied voltage and $\mathrm{V}_{\mathrm{CR}}$ is the threshold value to initiate the corona discharge [16]. In our case, the $\mathrm{V}$ is proportional to the altitude (noted $\mathrm{H}$ in meters) and the fact that the experimental current may be fit to with the one calculated by using $\mathrm{i}=\mathrm{C} \mathrm{H}(\mathrm{H}-65)$ by adjusting the value of $\mathrm{C}$ (see Fig 7-a) indicates that the collected current originated from a typical corona discharge.

The current value fluctuated but generally increased with the increase in altitude. When the gondola reached an altitude of $125 \mathrm{~m}$, the average current was $320 \mathrm{nA}$ for this experiment. This corresponds to a capacitor voltage of $32 \mathrm{~V}$. As the voltage probe is a divisor by 1000 , this means that the voltage displayed on the screen is $32 \mathrm{mV}$, thus comfortably measurable on the oscilloscope. As the capacitor was connected to ground, the tip was at a potential of $32 \mathrm{~V}$ in this case. This provides support for our claim in section 2 that compared to a potential of $12.5 \mathrm{kV}$, the tip potential is very close to that of the gondola.

The experiment was repeated more than 20 times at different moments in the day, and the same trend was observed, as shown in figure 7-b using the five records of them. However, for a given altitude, the current value changed from one ascent to another. The current depends on several parameters such as the electrical field between the Earth's surface and the troposphere (up to $125 \mathrm{~m}$ in altitude) at the instant of measurement. The distance between the tip and the gondola also plays an important role. When the point is closer to the gondola, the potential is disturbed by the presence of the gondola, which is grounded as indicated above. Because of wind, the distance between the tip and the gondola fluctuated considerably.

\subsection{Current measurement on a cycle of ascent/descent}

The balloon made several cycles of ascent/descent. Each cycle consisted of 2.6 minutes ascent, 2 minutes parking at $125 \mathrm{~m}$ and 3.5 minutes descent. The current as a function of time during a cycle is illustrated in figure 8 . The altitude as a function of time is represented by the dotted curve. When the altitude was $125 \mathrm{~m}$, the gondola moved constantly because of the wind, as did the tip with respect to the gondola. This is certainly the main reason for the fluctuation of the corona discharge current. During the descent, the current decreased and disappeared when the altitude was less than $66 \mathrm{~m}$, the same threshold value as that found during ascent.

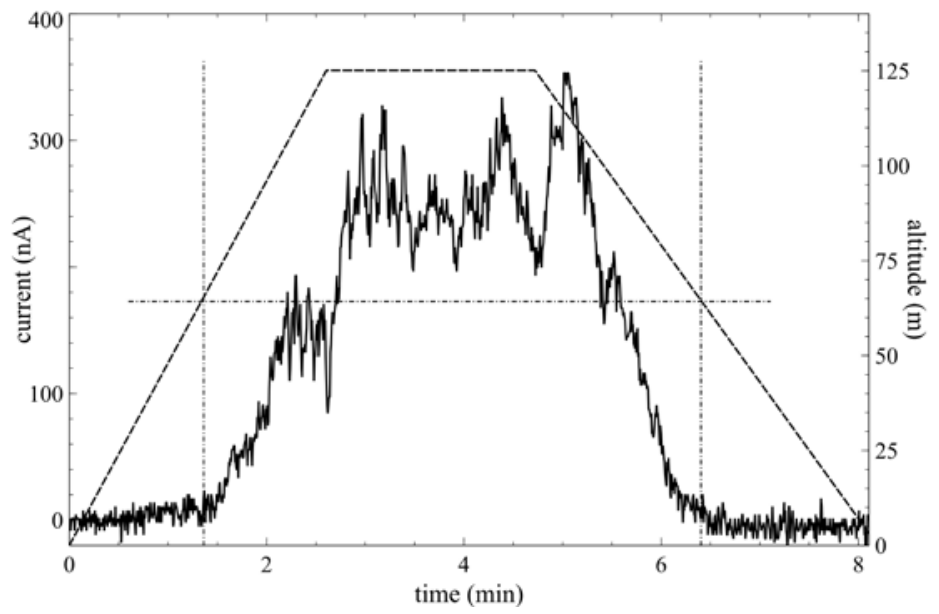

Fig. 8: Typical current and altitude as a function of time during a cycle. The thin dashed lines indicate the thresholds in altitude for corona discharge, both during the ascent and the descent. 


\subsection{Complementary measurements}

During the stationary time of the balloon at an altitude of $125 \mathrm{~m}$, a complementary experiment was done to demonstrate again the existence of a non-zero current. Indeed, if the voltage probe is not connected to the capacitor, the resistance $\mathrm{R}$ of the equivalent circuit in figure 3 is no longer present and there is no current diversion. In this case, the capacitor can be charged to a voltage well above $32 \mathrm{~V}$. To verify this, the capacitor was allowed to charge for 30 seconds without the high voltage probe, and then grounded electrical cable was placed in close proximity to the high potential terminal of the capacitor, which produced a spark before the two conductors made contact. This indicates that the voltage should be much larger than $32 \mathrm{~V}$, in other words, the capacitor was charged by a non-zero current.

In order to check that the real electrical circuit corresponded to the one in the figure 3 , a second high voltage probe was connected in parallel to the original voltage probe for an equivalent input impedance of $50 \mathrm{M} \Omega$, i.e., half that of the single probe. The voltage measured at the capacitor terminals was then half of that given by the single probe, thus giving the same value for the corona discharge current as shown in the figure 9 .

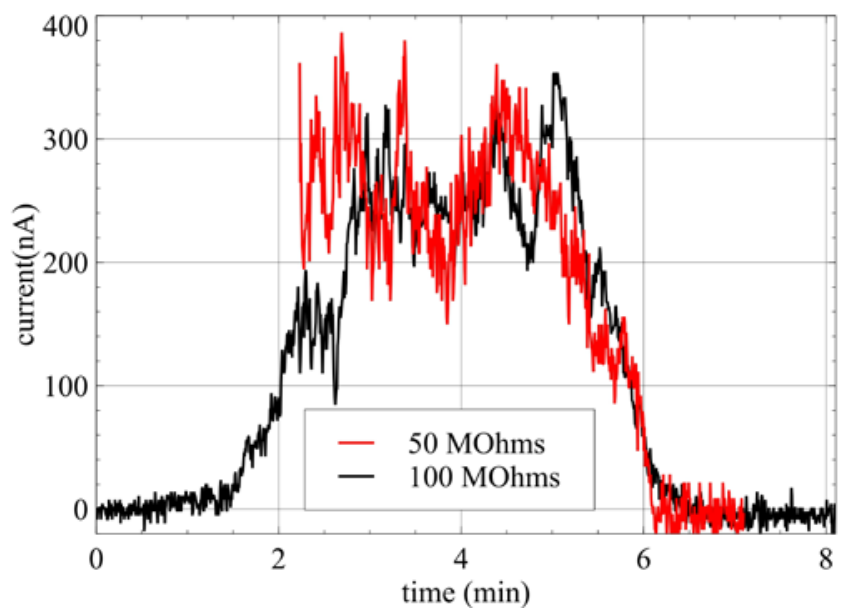

Fig. 9: Current as a function of time during a cycle. The current was measured with the circuit of the figure 3 with $\mathrm{R}$ value of $50 \mathrm{M} \Omega$ (red line) and $100 \mathrm{M} \Omega$ (black line) respectively.

Finally, the tip was replaced by a rounded tip conductor with a $2 \mathrm{~mm}$ radius of curvature. No measurable current was detected. This means that the electric field near the rounded electrode placed at an altitude of $125 \mathrm{~m}$ was not large enough to induce an observable corona discharge in our experimental conditions.

\subsection{Influence of an insulating surface near the point electrode}

In figure 1-b, a flag is schematically represented. The tip was placed near the flag which was close to the envelope of the balloon. Under these conditions, no current was detected. The flag and the tip were then moved to the same altitude as the gondola, far from the envelope of the balloon, and again, no current was detected. Then, the tip was placed 4 meters from the flag, and the current was again observed.

These observations can be explained as follows: an insulated object in the atmosphere is electrically charged until it reaches a potential balance with its surroundings, but the time to obtain the potential balance should be longer than tens of minutes due to the low rate of charge exchange between the flag and the surrounding air. 
In our tests, since the flag stays close to the ground much longer, the electric charges carried by the flag induce a low potential. Therefore, when the tip is placed close to the flag, it is in an unfavorable environment to produce a corona discharge.

If the flag is placed at $125 \mathrm{~m}$ for a long enough time, which remains to be determined, it is possible that a tip placed near the flag would have produced a corona discharge current. Unfortunately, we were unable to carry out such a test. If the insulating flag is replaced by a conductive object equipped with a potential equalizer such as a water-dropper for instance, it is likely that a tip placed close to this would rapidly produce a discharge current.

\section{CONCLUSION}

This study demonstrated that it is possible to get a natural high voltage due to the vertical potential gradient. This indirect observation was evidenced by the existence of a corona discharge without any high voltage power supply. From the point of view of physical observations, the following points can be noted:

1) As soon as a threshold was reached, a grounded point with a $25 \mu \mathrm{m}$ in radius of curvature and placed away from the gondola produced a corona discharge.

2) In fine weather, the collected current increased with altitude, and reached a value of about $320 \mathrm{nA}$ at $125 \mathrm{~m}$ in altitude.

3) The collected current fluctuated with time, wind and its distance from the gondola.

4) At an altitude of $125 \mathrm{~m}$, a grounded electrode with a $2 \mathrm{~mm}$ radius of curvature was unable to produce an observable corona discharge current.

5) On a time scale of a minute, a tip placed at $125 \mathrm{~m}$ cannot produce a current if it is placed near a large insulating object that has previously stayed a long time close to the ground.

\section{ACKNOWLEDGMENT}

We thank the staff of the Balloon of Paris for their help. We also thank the GDR SEEDS for the partial financial support.

\section{REFERENCES}

[1] Feynman R, Leighton R B and Sands M 1963. The Feynman Lecture on Physics (MA: AddisonWesley, Reading) Vol 2Chap. 9 'Electricity in atmosphere'.

[2] Chalmers J A 1967. Atmospheric Electricity 2nd Edn (Oxford: Pergamon)

[3] Kirkman J R and Chalmers J A 1957. Point discharge from an isolated point J. Atmosph. Terr. Phys 10 258-65

[4] Whipple F JW and Scrase F J 1936 Point discharge in the electric field of the earth Geophys. Mem. 68 1-20

[5] Chalmers J A and Mapleson WW 1955 Point discharge currents from a captive balloon J. Atm. Terr. Phys. 6 149-59

[6] Large MI and Pierce E T 1957 The dependence of point-discharge currents on wind as examined by a new experimental approach J. Atm. Terr. Phys. 10 251-7

[7] Chapman S 1958 Corona-point-discharge in wind and application to thunderclouds Recent Advances in Atmospheric Electricity, (London: Pergamon Press) pp 277-88

[8] Chalmers J A 1965 The effect of wind on point-discharge pulses J. Atm. Terr. Phys. 27 1037-8 
[9] Gernsback H 1922 Power from the Air (I), Science and Invention 9 10. Gernsback H 1922 Power from the Air (II), Science\&Invention 9 1006-7

[10] Grandics P 2008ANelectric power converter for extraction of atmospheric electric energyWO/2008/005628

[11] Grandics P 2010 Pyramid Electric Generator US2010/0207399

[12] Ogram ME 2012 Atmospheric Energy Collection US2012/0286623

[13] Renard J-B et al 2015 LOAC: a small aerosol optical counter/sizer for ground-based and balloon measurements of the size distribution and nature of atmospheric particles-II: first results from balloon and unmanned aerial vehicle flights Atmos. Meas. Tech. Discuss. 8, 1261-99

[14] Trichel GW 1938 The mechanism of the positive point-to-plane corona in air at atmospheric pressure Phys. Rev. 55 382-90

[15] Raizer Y P 1997 Gaz Discharge Physics, Corrected 2nd printing (Heidelberg, New York: Spinger-Verlag Berlin) 3-540-1946-2

[16] Lama WL and Gallo Cf 1974 Systematic study of the electrical characteristics of the 'Trichel' current pulses from negative needle-toplane coronas JAP 45 103-13 